\title{
Inbreeding Depression
}

Francisco Ceballos ${ }^{1}$

1 University of Witwaters rand

The reduction in evolutionary fitness of a population or individual due to the presence of increased homozyg osity arising from inbreeding. Values of traits related to fitness, such as fertility, are reduced. 